SCIPP 10/07

\title{
Axions in the Landscape and String Theory
}

\author{
Michael Dine ${ }^{(a)}$, Guido Festuccia ${ }^{(b)}$, John Kehayias ${ }^{(a)}$ and Weitao Wu ${ }^{(a)}$ \\ (a) Santa Cruz Institute for Particle Physics and \\ Department of Physics, Santa Cruz CA 95064 \\ (b) School of Natural Sciences, Institute for Advanced Study \\ Einstein Drive, Princeton, NJ 08540
}

\begin{abstract}
While axions seem ubiquitous in critical string theories, whether they might survive in any string theoretic description of nature is a difficult question. With some mild assumptions, one can frame the issues in the case that there is an approximate supersymmetry below the underlying string scale. The problem of axions is then closely tied to the question of how moduli are fixed. We consider, from this viewpoint, the possibility that supersymmetry is broken at an intermediate scale, as in "gravity mediation," at a low scale, as in gauge mediation, and at a very high scale, to model the possibility that there is no low energy supersymmetry. Putative mechanisms for moduli fixing can then be systematically classified, and at least for intermediate and high scale breaking, light axions appear plausible. In the course of this work, we are lead to consider aspects of moduli fixing and supersymmetry breaking, and we revisit the possibility of very large extra dimensions.
\end{abstract}




\section{Why String Theory Seems a Promising Setting to Solve the Strong CP Problem}

¿From the beginning, the Peccei-Quinn (PQ) solution to the strong CP problem had a troubling aspect: why should the underlying theory obey a global symmetry to an extremely good approximation. Indeed, the approximation must be so good that any operator which would violate the symmetry must be of very high dimension, even if its coefficient is scaled by inverse powers of the Planck scale. As a result, to tackle the problem one requires a theory where such ultraviolet questions can be meaningfully addressed. String theory does appear to provide an answer: there are often scalar fields, which, in perturbation theory have no potential, and which couple to $F \widetilde{F}$ of candidate standard model gauge fields [1]. But, while suggestive, assessing the viability of the axion in string theory, and more generally the axion itself (viewing string theory as a model for the axion phenomenon) requires understanding how supersymmetry is broken and moduli are fixed. If the non-perturbative effects which break supersymmetry also break the Peccei-Quinn symmetry, then the symmetry cannot resolve the strong CP problem. In the KKLT proposal [2], the most complete proposal for how moduli might be fixed in string theory, there is one seemingly natural axion candidate, the pseudoscalar field which is the partner of the single Kähler modulus. But this field gets a large mass, larger than the scale of supersymmetry breaking. Indeed, the full supermultiplet gains a mass which is approximately supersymmetric. This has lead to pessimism about obtaining QCD axions in a landscape [3, 4]. In this paper, we revisit this issue, and discover that there are circumstances where axions of sufficient quality to solve the strong CP problem are plausible.

In order to assess the likelihood of light axions in string theory, with the present (limited) state of our understanding, it is necessary to make some assumptions about the underlying symmetries and dynamics. Probably the most important of these is the scale of supersymmetry breaking. We will focus here on three possibilities: intermediate scale breaking (ISB) of supersymmetry, as in "gravity mediation" $\left(M_{\text {int }} \approx \sqrt{\mathrm{TeVM} M_{p}}\right)$, lower scale of supersymmetry breaking, as in gauge mediation, and a scale much higher than the intermediate scale, as a model for a situation with no low energy supersymmetry.

In all three cases, if moduli are somehow fixed and are not extremely light, the partners of any very light axions must be fixed by supersymmetry breaking dynamics. So we wish to investigate whether moduli can indeed be fixed in such a fashion, while leaving over a Peccei-Quinn shift symmetry of high quality.

The known axion candidates in string theory respect certain shift symmetries. Given our assumption that supersymmetry survives below the fundamental scale, these lie in chiral multiplets, which we will refer to as "axion multiplets:"

$$
\tau_{i} \rightarrow \tau_{i}+2 \pi i n_{i}
$$

We choose here to take the axion to be the imaginary part of $\tau_{i}$,

$$
\tau_{i}=s_{i}+i a_{i}
$$


We will sometimes refer to $\tau_{i}$ and/or $s_{i}$ as "axionic supermultiplets."

If generated by short distance (e.g. string scale instanton) effects and if $s_{i}$ is large, these necessarily appear in any superpotential through terms of the form

$$
W_{\tau}=e^{-m_{i} \tau_{i}},
$$

for some integers $m_{i}$. We will assume that the axion decay constants are of order the underlying fundamental scale, $M$, which we will imagine ranges from $10^{15} \mathrm{GeV}$ to the Planck scale (though at one point we will relax this, when we consider the large extra dimension scenario of [5]). We will assume that the continuous shift symmetry is broken by high scale "instanton" effects of order $e^{-n \tau}$, with $n$ an integer. The requirement that the Peccei-Quinn symmetry is of high quality is then a constraint on the $s_{i}$ 's. To qualify as a QCD axion, for example, assuming that the fundamental scale is $10^{16} \mathrm{GeV}$, gives $s>180$ or so. We will see, following ideas of $[6,7,8]$ that there are quite plausible mechanisms which could account for the required $s_{i}$ for multiple axions. The low energy dynamics of these moduli can be analyzed simply within the framework of low energy effective field theory. Moreover, these additional moduli may themselves be the source of supersymmetry breaking and small, positive cosmological constant (c.c.). The main difficulties with this framework are the familiar ones of ISB: flavor, the cosmological moduli problem [9, 10], and the overshoot (or Brustein-Steinhardt [11]) problem. The framework which we develop to study the dynamics of such light axions allows a quite simple analysis of the large extra dimension scenario of [5]. We will see that the scenario is prone to a variety of instabilities, but, as has been discussed in the literature on this subject, it remains quite plausible that such stationary points exist. Whether they are particularly common in a landscape is another matter, about which we can (and will) only speculate.

An alternative possibility is that the Peccei-Quinn symmetry arises as an accidental, approximate symmetry, linearly realized at scales well below $M$. We will argue, following [12], that in the case of low energy supersymmetry breaking, cosmology probably necessitates such a picture. The most likely way such a symmetry might arise would seem to be the presence of discrete $R$ (and possibly other) symmetries. This scenario does not suffer from flavor problems, or the usual cosmological moduli problem. The question, in this context, is why the quality of the Peccei-Quinn symmetry is so high.

In this note, we will explore more generally the question of Peccei-Quinn symmetries in an underlying landscape. In line with our remarks above, we will consider various possible situations:

1. Supersymmetry broken at an intermediate scale (ISB).

2. Supersymmetry broken at a low scale, as in gauge mediation.

3. Supersymmetry broken at a high scale, in part in order to model the absence of supersymmetry.

We will see that the possibilities for moduli fixing, with large $s_{i}$, can be simply classified. They include two mechanisms which fix a linear combination of the $\tau_{i}$ 's, while preserving an approximate supersymmetry: 
1. The small $W_{0}$ mechanism $[6,8]$. Here $W_{0}$ is a constant term in the superpotential. In this case, a linear combination of the $s_{i}$ 's is fixed in an approximately supersymmetric fashion, with the large value of $s_{i}$ being related to the small value of $W_{0}$. At low energies. one linear combination of $\tau_{i}$ 's can be integrated out, leaving a theory with a set of $\tau_{i}$ 's, a constant superpotential, and with some Kähler potential. The (generalized) KKLT scenario fits within this framework.

2. The "Racetrack:" Here, the hierarchy arises from a delicate balance between different exponentials in the superpotential. Again, some linear combination of moduli are fixed, leaving other light fields which can play the role of the QCD axion (and additional axions) [7, 8]. This theory, again, is described by a constant superpotential and some Kähler potential.

Alternatively, all of the $\tau_{i}$ 's can be fixed simultaneously with supersymmetry breaking. This falls within the class of stabilization mechanisms known as "Kähler stabilization."

In the next section, we consider the small $W_{0}$ scenario. We review why there is no light axion in the case of a single light modulus. We explain that in the case of multiple light moduli, the effective low energy theory is very simple, and it can readily be seen that:

1. Under plausible conditions, all of the $s_{i}$ moduli are stabilized by supersymmetry breaking.

2. There can be multiple light axions, including the QCD axion, with the required high quality.

3. There is a quite real possibility of breaking supersymmetry through the dynamics of the axionic moduli, without additional fields.

In Section 3, we discuss racetrack and Kähler stabilization. Like the small $W_{0}$ case, in the case of the racetrack, there is typically one modulus with mass large compared to $m_{3 / 2}$, while others have masses, again, of order $m_{3 / 2}$. In the case of Kähler stabilization, all of the moduli have masses of order $m_{3 / 2}$.

In Section 4, we discuss the question of whether supersymmetry can be broken by the dynamics of the Kähler moduli themselves, without invoking antibranes or low energy dynamics on branes, or other phenomena. We show that this is a logical possibility. In Section 5, we show that the Large Extra Dimension scenario of [5] fits naturally in this framework. The appearance of exponentially large volumes is readily understood, as well as potential instabilities, which we discuss. In Section 6, we briefly review two well-known issues associated with models of intermediate scale supersymmetry breaking: flavor and cosmological moduli, and their relevance to the axion solution of the strong CP problem.

In Section 7, we discuss axions in the frameworks of low energy gauge mediation and high scale supersymmetry breaking. Finally, in our conclusions, we provide a scorecard for different settings of the Peccei-Quinn solution of the strong CP problem. 


\section{Axions in the Small $W_{0}$ Scenario with Multiple Kähler Moduli}

The KKLT proposal is a well-known realization of the small $W_{0}$ scenario. Independent of the microscopic details, it can be summarized in a very simple low energy Lagrangian. At scales below the fundamental scale, there is a single chiral field, $\rho$, with superpotential

$$
W=W_{0}+\alpha e^{-\rho / b}
$$

The Kähler potential is:

$$
K=-3 \ln \left(\rho+\rho^{\dagger}\right)
$$

$W_{0}$ is a constant (as is $\alpha$ ). The most important aspect of the KKLT analysis is the claim that $W_{0}$ can be extremely small; this becomes the small parameter which permits self-consistent approximations (it accounts, in particular, for large $\rho$ ). With this assumption one can solve sequentially for a stationary point of the potential. First, study the equation for a supersymmetric minimum:

$$
\frac{\partial W}{\partial \rho}+\frac{\partial K}{\partial \rho} W=0 .
$$

The minimum occurs for

$$
\rho \sim-b \ln \left|W_{0}\right|+b \log \left(-\log \left|W_{0}\right|\right) .
$$

At the minimum, the $W_{0}$ term in the superpotential dominates by a power of $\rho$.

The low energy Lagrangian is a supergravity Lagrangian with superpotential

$$
W \approx W_{0} .
$$

The mass of the components of $\rho$ is of order

$$
m_{\rho}^{2} \sim e^{K}\left|W_{0}\right|^{2} \rho^{2} .
$$

In particular, if supersymmetry is broken in some fashion (e.g. anti-D branes, as suggested in KKLT, or some low energy dynamics, perhaps associated with fields on branes), the mass of the modulus multiplet is larger than the gravitino mass by a factor of $\rho$; this justifies solving the equation for $\rho$ first.

If this is all there is to the low energy theory, supersymmetry is unbroken and the cosmological constant is negative. KKLT, and subsequently others, have provided a variety of scenarios for how supersymmetry might be broken, and for the origin of a substantial positive contribution to the vacuum energy. The most popular of these involves anti-D3 branes $^{1}$, but one can alternatively imagine that there are simply some additional interactions in the low energy theory which break supersymmetry [13, 14]. Adopting such a model, we can represent this through the presence of a field, $Z$, with a non-zero $F$

\footnotetext{
${ }^{1}$ As often formulated, this is puzzling, as it is not clear how supersymmetry is spontaneously broken; additional fields are necessarily required, e.g. to provide the longitudinal component of the gravitino. The resolution may lie in the fact that the effective theory is not actually four dimensional, due to an infinite tower of additional fields.
} 
component. Depending on the underlying details, this $F$ component, and the Kähler potential, may exhibit non-trivial $\rho$ dependence. For the moment, we will make no assumptions about the field $Z$; in Section 4, we will explore the possibility that this field is itself one of the Kähler moduli.

In this theory, the fields in the $\rho$ multiplet all have mass of order $\rho m_{3 / 2}$, and there is typically an additional multiplet whose components have masses of order $m_{3 / 2}$ (often including a pseudomodulus). Whatever the detailed mechanism of supersymmetry breaking, the $\rho$ multiplet, including the pseudoscalar candidate axion, has an approximately supersymmetric spectrum, and the axion is not suitable for solving the strong CP problem.

Thinking about the problem more generally, it is clearly necessary that the partner of any would-be axion gain mass only as a consequence of supersymmetry breaking [12]; otherwise the axion is massive. This requires that the superpotential, to a high degree of accuracy, be a function of only one linear combination of moduli,

$$
W=W_{0}+e^{-\tau / b}
$$

(we will assume $b>1$, as in gaugino condensation); here $\tau=\sum n_{i} \tau_{i}$ (for IIB orientifolds of Calabi-Yau manifolds, the $\tau_{i}$ 's would represent additional Kähler moduli).

At the same time, it is necessary that all of the moduli, $\tau_{i}$, be fixed, in a fashion such that $e^{-\tau_{i}} \ll$ $e^{-\tau / b}$, More precisely, to obtain an axion suitable to solve the strong CP problem (and more generally, to give rise to the axiverse of [15]), one requires that

$$
W=W_{0}+e^{-\tau / b}+A_{i} e^{-\tau_{i}}+\ldots
$$

$\tau$ is then fixed along the lines of KKLT, and the (real parts) of the other Kähler moduli are all fixed as a result of the structure of the Kähler potential, in such a way that all additional terms in the superpotential extremely small.

This may seem non-generic, and this was the point of view of [12]. But it is actually not difficult to see how this might occur[6,8]. For the superpotential of eq. (8), one can solve the equation

$$
D_{\tau} W=0
$$

with

$$
\tau \approx-b \log \left(\left|W_{0}\right|\right)
$$

If the Kähler metric for $\tau$ vanishes at large $\tau$, as is typical of familiar supergravity and string constructions, then $\tau$ is heavy, and can be integrated out. The remaining light fields, $\tau_{i}$, are described by a theory with some Kähler potential (a function of $\left(\tau_{i}+\tau_{i}^{\dagger}\right)$ ) and a constant superpotential. Suppose that the Kähler potential has a stationary point at $\tau_{i}^{0}$,

$$
\partial_{i} K=0
$$


With no other fields or dynamics, this point would correspond to a supersymmetric, AdS vacuum. If there are additional dynamics which break supersymmetry (as assumed, for example, in the KKLT model) giving small cosmological constant, then, quite generally, the masses of the remaining $\tau_{i}$ fields receive a contribution to their masses-squared equal to $m_{3 / 2}^{2}$ from their mutual interactions. Consider the potential:

$$
V_{\left(\tau_{i}+\tau_{i}^{\dagger}\right)}=e^{K}\left(\left|W_{0}\right|^{2} K_{i} K^{i j} K_{j}-3\left|W_{0}\right|^{2}\right),
$$

where we have noted that it is only the combination $\left(\tau_{i}+\tau_{i}^{\dagger}\right)$ that appears in $K$ and thus the potential. Differentiating twice and using the fact that the $K_{i}$ 's vanish at the stationary point, indeed yields $-K_{i j}\left|W_{0}\right|^{2} e^{K\left(\tau_{i}^{0}+\tau_{i}^{0 \dagger}\right)}$.

Whether this is the entire contribution to the masses of these moduli depends on the nature of supersymmetry breaking. Suppose, for example, that supersymmetry is broken by the $F$ component of a chiral field, $X$, through an additional term in the superpotential $f X$. Then, depending on the structure of the Kähler potential for $X$, there can be additional contributions to the masses of the $\tau_{i}$ fields. Terms such as $X^{\dagger} X /\left(\tau_{i}+\tau_{i}^{\dagger}\right)$ respect the shift symmetry, and contribute to the masses, potentially with either sign, an amount of order $m_{3 / 2}$. So whether these points are actually local minima of the potential depends on such details.

The procedure of integrating out the heavy modulus can be illustrated by a simple example. We will use the language of the IIB theory. First, suppose we consider a version of KKLT with two Kähler moduli, $\rho_{1}$ and $\rho_{2}$, and with superpotential

$$
W=W_{0}-A e^{-\frac{\rho_{1}+\rho_{2}}{b}}+f X
$$

and Kähler potential:

$$
K=-\frac{3}{2} \log \left(\left(\rho_{1}+\rho_{1}^{\dagger}\right)\left(\rho_{2}+\rho_{2}^{\dagger}\right)\right)+K\left(X, X^{\dagger}\right) .
$$

Here, for simplicity, $K\left(X, X^{\dagger}\right)$ is such that it gives rise to a minimum for $X$ near the origin, e.g.

$$
K=X^{\dagger} X+\frac{1}{m^{2}} X^{\dagger} X X^{\dagger} X
$$

Such a superpotential could arise in the presence of gaugino condensation in a sector with gauge coupling

$$
\rho_{0}=\rho_{1}+\rho_{2}
$$

and $\beta$-function $b / 3$. As in the original KKLT model, this model has an approximate, supersymmetric stationary point at

$$
\rho_{1}=\rho_{2}=x \approx \frac{b}{2} \log \left(\left|W_{0}\right|\right) .
$$

$\rho_{0}$ has mass-squared of order $\rho_{0}^{2}\left|W_{0}\right|^{2}$, and can be integrated out, leaving an effective theory for

$$
\Psi=\rho_{1}-\rho_{2}
$$


with Kähler potential and superpotential

$$
K=\frac{1}{\rho_{0}^{2}}\left(\Psi+\Psi^{\dagger}\right)^{2}+\ldots+K\left(X, X^{\dagger}\right) \quad W=W_{0}+f X .
$$

For suitable adjustment of $W_{0}$ and $f$, as suggested in [2,16], the cosmological constant can be arbitrarily small. In this case, the minimum of the potential for Re $\Psi$ lies at the origin, and the $\Psi$ field has mass of order $m_{3 / 2}$. It is easy to consider more realistic Kähler potentials and follow through the same procedure.

How light is the axion? High energy non-perturbative effects are of order $e^{-\rho_{0}}$. So if $b$ is of order, say 4 , and

$$
e^{-\rho_{0} / b} M_{p}=100 \mathrm{TeV}
$$

then

$$
e^{-\rho_{0}}=10^{-52}
$$

This gives an axion of high enough "quality" [12] to account for the axion of QCD. Of course, factors of two in the exponent can make an appreciable difference in one direction or the other.

It is natural, in this low energy picture, to ask whether the field $X$ is necessary; could the low energy theory for some set of fields, $\Psi_{i}$, with constant superpotential, be responsible for supersymmetry breaking? We will explore this question in Section 4.

\section{Other Stabilization Mechanisms: Racetrack Models and Kähler Stabilization}

The essential ingredients in our analysis above were:

1. The presence, at some low energy scale, of multiple axion superfields, $\tau_{i}=s_{i}+i a_{i}$, invariant under a discrete shift symmetry.

2. As a consequence of (1), for large $s_{i}$, holomorphic quantities depend on $\tau_{i}$ as $e^{-n_{i} \tau_{i}}$. It is necessary that $\rho_{i}$ be large if the corresponding axion is to be light.

The existence of this small parameter for the would-be axion is particularly important. In the KKLT scenario, the large size of $\rho$ is correlated with the small parameter $W_{0}$; the large value of some linear combination of $\rho_{i}$ 's is determined by supersymmetric dynamics; supersymmetry breaking determines the relative values of the different moduli. We will take this as our definition of the KKLT scenario (in this sense, it applies to the proposals in $[7,8])$.

There are other ways we might imagine a small parameter could come about, which would allow for the possibility of light axions. First, there is the possibility that one obtains a large value of all of the $\rho_{i}$ 's 
through dynamics which break supersymmetry. This would be a realization of "Kähler stabilization" [17]. In this context, there would again be a superpotential which is a function of a linear combination of the moduli. The Kähler potential of the theory would give rise to supersymmetry breaking and a large expectation value for this modulus; each of the other moduli would similarly be large. The spirit of the Kähler stabilization hypothesis is that, despite the fact that the theory is not weakly coupled, certain holomorphic quantities, the factors $e^{-\rho_{i}}$ in this case, are extremely small. Whether this actually happens in string theory is an open question.

Another possibility is that the low energy theory for the $\tau_{i}$ 's (the theory obtained after integrating out the complex structure moduli in the case of KKLT) contains no constant in the superpotential, perhaps due to an unbroken, discrete $R$ symmetry. The superpotential for $X$ is would be generated by non-perturbative effects, e.g. as in retrofitted models $[18,19]$. These effects would break the $R$ symmetry, generating the requisite expectation value of the superpotential. Fixing of the Kähler moduli, with remaining axions, follows as in the previous section.

So arguably the appearance of axions in such a picture is robust. So far, we have assumed that $m_{3 / 2} \sim \mathrm{TeV}$. If we are not wedded to supersymmetry as a solution of the hierarchy problem, we can consider the case of much larger $m_{3 / 2}$; then we have a model for axions without low energy supersymmetry. What is mainly required is that $e^{-\rho_{0} / b}$ still be hierarchically small, so that plausible powers of this parameter can account for the quality of the QCD axion. Indeed, well known cosmological considerations $[9,10]$ require that the scale be $50 \mathrm{TeV}$ or larger; we will review these in Section 6.

Lower $m_{3 / 2}$ would arise, for example, in gauge mediation. Here it is problematic that the partner of the axion must be fixed by supersymmetry breaking dynamics. If it's interactions are Planck suppressed, this particle leads to an untenable cosmology. To obtain axions, one either requires that the scale of interactions between the axion multiplet and the hidden sector is significantly lower than $M_{p}$, or that there are no light moduli, and the Peccei-Quinn symmetry is linearly realized for some range of scales in the low energy theory. We will discuss these issues in Section 7.

\section{Supersymmetry Breaking With Multiple Moduli}

We have seen in the previous section that if there are additional light fields which break supersymmetry, we can readily understand how all of the Kähler moduli are fixed in a way that non-perturbative corrections to the axion potentials are small. In this section, we discuss the possibility that the additional Kähler moduli are themselves responsible for supersymmetry breaking. In general, we are dealing with a theory with a constant superpotential and some Kähler potential. It is easy to see that it is possible, in principle, for supersymmetry to be broken in this situation.

Near any would-be stationary point of the potential, we still expect to find one heavy field, $\Phi$, and several light fields, $\rho_{i}$. These fields are distinguished by the invariance of the theory under discrete 
shift symmetries. Integrating out $\Phi$, the low energy theory will again be described by a constant superpotential, $W_{0}$, and a Kähler potential for $\rho_{i}$. We still have good, approximate shift symmetries,

$$
\rho_{i} \rightarrow \rho_{i}+2 \pi i n_{i}
$$

We can ask whether this theory can break supersymmetry. In fact, under rather general conditions, this low energy theory does exhibit supersymmetry preserving and supersymmetry breaking stationary points. The supersymmetry preserving points are AdS, and satisfy the Breitenlohner-Freedman bound [20, 21]. The supersymmetry breaking points have lower (i.e. more negative) cosmological constant. So if these states are obtained in any sort of systematic approximation, additional dynamics are required to account for a small, positive cosmological constant.

If the function $K\left(\rho_{i}+\rho_{i}^{\dagger}\right)$ has a stationary point:

$$
\partial_{i} K=0 \forall i ; \rho_{i}=\rho_{i}^{0}
$$

then supersymmetry is unbroken at this point, with negative potential,

$$
V_{0}=-3 e^{K\left(\rho^{0}\right)}\left|W_{0}\right|^{2}
$$

while each of the $\left(\rho_{i}+\rho_{i}^{\dagger}\right)$ 's are tachyonic, with

$$
m^{2}=-2 e^{K\left(\rho^{0}\right)}\left|W_{0}\right|^{2} .
$$

The Breitenlohner-Freedman bound is satisfied

$$
-\frac{9}{4} \leq m^{2} R^{2}=-2
$$

so these configurations describe stable AdS vacua. However, because the curvature of the potential is negative at this point, and because for large $\rho_{i}$ the potential typically tends to zero, we expect that the potential exhibits AdS supersymmetry breaking solutions.

\subsection{Simple Models which Break Supersymmetry}

This is illustrated by a simple example (we will also discuss the large volume solutions of [5] in Section 5). Rather than consider a Kähler potential with logarithmic behavior at $\infty$, take

$$
K=\frac{1}{2}\left(\rho+\rho^{\dagger}\right)^{2} .
$$

This admits a supersymmetric solution at $\left(\rho+\rho^{\dagger}\right)=0$, and a non-supersymmetric solution at

$$
\left(\rho+\rho^{\dagger}\right)= \pm 1 \text {. }
$$

The non-supersymmetric solution, indeed, has lower energy. 
Typically, when reliable computations are possible, this is problematic; the supersymmetry-breaking solutions are AdS. So additional dynamics are required to account for the observed small, positive cosmological constant.

But it is not at all clear that the underlying microscopic theory should be weakly coupled, and even if it is, its Kähler potential might be more complicated than we have contemplated above. We can ask whether it is logically possible, in a theory with constant $W$, to have broken supersymmetry and vanishing cosmological constant. In fact it is, as can be seen by considering a theory with a single scalar field, $\psi$, invariant under shifts.

We will suppose that the would-be minimum lies at $\psi=0$, and expand the Kähler potential about this point:

$$
K\left(\Psi+\Psi^{\dagger}\right) \equiv K(\psi)=k_{0}+k_{1} \psi+\frac{k_{2}}{2} \psi^{2}+\frac{k_{3}}{3} \psi^{3}+\frac{k_{4}}{4} \psi^{4} .
$$

We then require that the constants $k_{i}$ satisfy the following conditions:

1. The potential has a stationary point at $\psi=0$.

2. The potential is a minimum at $\psi=0$.

3. The kinetic terms for $\psi$ have a sensible sign at $\psi=0: k_{2}>0$.

4. Supersymmetry is broken at $\psi=0: k_{1} \neq 0$.

5. The cosmological constant is (nearly) zero at $\psi=0: k_{1}^{2}=3 k_{2}$.

It is easy to see that these conditions can be simultaneously satisfied.

\section{The Exponentially Large Volume Scenario}

The authors of [5] have put forth a scenario for the flux landscape in which the volume is "exponentially large." We will see that their scenario can be analyzed within the framework we have put forward here. There is a parameter, $W_{0}$ (which should be small, but need not be extremely small). Integrating out one modulus supersymmetrically leads to a low energy theory for the remaining moduli with constant superpotential and a particular Kähler potential. The leading terms in the potential, for this particular Kähler potential, cancel, leaving terms which vary logarithmically with the light modulus (this logarithmic variation is just the logarithmic modulus dependence familiar in KKLT). These terms are of the same order, for large values of the modulus, as the first subleading $\alpha^{\prime}$ corrections. The competition of these terms then allows for exponentially large solutions. The controlling, small parameter for their analysis, we will see, is $g_{s}$, the string coupling. In this setup, it is easy to understand why a modulus exponential in $1 / g_{s}$ naturally arises in the leading order analysis. It is also clear that this solution is potentially 
unstable. It is crucial to understand the form of stringy perturbative corrections to the Kähler metric for various fields. Some work has been done on this question [22, 23], and further investigations will be reported elsewhere [24].

Without reviewing all of the details of the large dimension models, suffice it to say that, like the models considered here, there are multiple Kähler moduli (in the examples they analyze in detail, 2). Following their notation, we will refer to these as $\tau_{4}, \tau_{5}$. The Kähler potential has a different form than that we have studied up to now:

$$
K=-2 \ln \left(\mathcal{V}+\frac{\xi}{2 g_{s}}\right)
$$

where

$$
\mathcal{V}=\tau_{5}^{3 / 2}-\tau_{4}^{3 / 2} \approx \tau_{5}^{3 / 2}\left(1-\left(\frac{\tau_{4}}{\tau_{5}}\right)^{3 / 2}\right)
$$

and where $\xi$ is a numerical constant. The superpotential can be taken to be (our notation is not identical to that of [5], and our form differs slightly, by redefinitions of fields).

$$
W=W_{0}+A e^{-\rho_{4}} .
$$

Here $\rho_{4}=\tau_{4}+i a$, i.e. $\rho_{4}$ is the superfield whose lowest component is $\tau_{4}$ plus an additional axion field.

Now, as in our previous analyses, $\tau_{4}$ is the heavy field, and we should integrate it out, solving its equations of motion. The equation $D_{\tau_{4}} W=0$ gives:

$$
\tau_{4}\left[\tau_{5}\right] \approx-\ln \left(W_{0} / \tau_{5}^{3 / 2}\right) .
$$

$\tau_{5}$ is the light field. To leading order in $1 / \tau_{5}$, the Kähler potential for $\tau_{5}$ is:

$$
K=-3 \ln \left(\tau_{5}\right)+2 \frac{\tau_{4}\left[\tau_{5}\right]^{3 / 2}}{\tau_{5}^{3 / 2}}-\frac{\xi}{g_{s} \tau_{5}^{3 / 2}} .
$$

where we have explicitly indicated that $\tau_{4}$ should be thought of, here, as a function of $\tau_{5}$. The superpotential for $\tau_{5}$ is approximately $W_{0}$, as in our earlier examples.

Because of the so-called "no-scale structure" of the Kähler potential, the leading terms in the potential, of order $m_{3 / 2}^{2} M_{p}^{2}=\frac{1}{\tau_{5}^{3}} \frac{\left|W_{0}\right|^{2}}{M_{p}^{2}}$ cancel. The next order terms, generated by the second and third terms in eq. (33) each behave as $\tau_{5}^{-9 / 2}$, up to logarithms. More precisely the potential behaves as a function of powers of $\log \left(\tau_{5}\right)$ times $\tau_{5}^{-9 / 2}$. It is this feature which leads to a stationary point at exponentially large $\tau_{5}$.

In the approximation that $\tau_{4}$ is large, the calculation of the potential greatly simplifies. In particular, in taking derivatives of $K$, terms obtained by differentiating $\tau_{4}$ are suppressed.

$$
K^{\prime} \approx-\frac{3}{\tau_{5}}\left(1+\frac{\tau_{4}^{3 / 2}}{\tau_{5}^{3 / 2}}-\frac{1}{2} \frac{\xi}{g_{s} \tau_{5}^{3 / 2}}\right)
$$




$$
\left(K^{\prime \prime}\right)^{-1} \approx \frac{\tau_{5}^{2}}{3}\left(1-\frac{5}{2} \frac{\tau_{4}^{3 / 2}}{\tau_{5}^{3 / 2}}+\frac{5}{4} \frac{\xi}{g_{s} \tau_{5}^{3 / 2}}\right) .
$$

Correspondingly, the potential is approximately:

$$
V=-3|W|^{2}\left(\frac{1}{2} \frac{\tau_{4}^{3 / 2}}{\tau_{5}^{3 / 2}}-\frac{1}{4} \frac{\xi}{g_{s} \tau_{5}^{3 / 2}}\right) \frac{1}{\tau_{5}^{3}}
$$

Since both terms exhibit the same $\tau_{5}^{-9 / 2}$ power law dependence, the competition of the logarithmic dependence of the first term and the (large, for small $g_{s}$ ) constant in the second term gives rise to an exponentially large solution:

$$
\tau_{5} \approx e^{\left(\xi / g_{s}\right)^{2 / 3}}
$$

This is the result found in [5].

There are now several issues. First, the result is crucially dependent on the structure of the leading terms in the Kähler potential. Corrections in $g_{s}$ might spoil this. For a smooth manifold, one might expect these corrections to be suppressed by powers of the compactification radius. This follows from the fact that at distances small compared to $R$, the theory is ten dimensional, so contributions to loops from high Kaluza-Klein modes would be essentially ten dimensional, in which case supersymmetry forbids corrections to kinetic terms. Still, the power must be rather large, and one might worry, in addition, that an orientifold is not a smooth manifold. These questions have been studied in [22] and [23], who argue that, while such corrections are present, they are such that the first subleading corrections to the potential cancel as well, and the result is stable. This question will be studied further in [24].

A second issue is that the cosmological constant is negative in these states ${ }^{2}$. By assumption, the calculations are reliable in this limit, so additional dynamics are required in order to understand a small, positive vacuum energy. These have the potential to further destabilize the vacuum. As a model, add a chiral field $X$ to the theory, with superpotential

$$
\delta W=f X
$$

and Kähler potential adjusted so that the $X$ potential has a stationary point at the origin. We require that $f$ be such that it cancel the vacuum energy $-V_{0}$. This requires

$$
\left|f^{2}\right| \sim \frac{\left|W_{0}\right|^{2} \log \left(\tau_{5}\right)^{\frac{1}{2}}}{\left(\tau_{5}^{0}\right)^{3 / 2}}
$$

where $\tau_{5}^{0}$ is the value of $\tau_{5}$ near the minimum.

For such a value of $f$, this is a small perturbation on the $\tau_{5}$ potential and the value of $\tau_{5}$ at the minimum $\tau_{5}^{0}$ changes by an order one multiplicative constant with respect to the $f=0$ case. The

\footnotetext{
${ }^{2}$ Note that if the cosmological constant were positive, this would be particularly problematic, since by assumption corrections are small. Additional supersymmetry breaking dynamics might be expected to add only additional positive contributions, so some more significant modification of this structure would be required.
} 
viability of the large dimension solution critically depends on this tuning of $f^{2}$. If $f^{2}$ were too large, say by a factor of 100 , the "corrections" to the $\tau_{5}$ potential would overwhelm those considered in [5]. One would be driven back to the small $W_{0}$ (KKLT) scenario. In a landscape, then the question might be something like: how common are exponentially large dimensions (requiring tuning of $f^{2}$ ) vs. very small $W_{0} ?$

Even if we suppose that the cosmological constant can be explained while still obtaining an exponentially large volume solution, there are other issues. Examining the potential and the kinetic terms, we see that the mass of $\tau_{5}$ is small compared to $m_{3 / 2}$;

$$
m_{\tau_{5}}^{2} \approx m_{3 / 2}^{2} \tau_{5}^{-3 / 2}
$$

due, again, to the "no scale" cancellations. As has been widely discussed in the literature, this is potentially problematic cosmologically. To deal with this issue, it has been suggested [25] that the scale, $m_{3 / 2}$ is large, and that matter fields are light (by powers of $\tau_{5}$ compared to $m_{3 / 2}$ ) due to a no scale structure.

It is important that this feature survive at the quantum level. Examination of low energy diagrams suggests that this hierarchy can be stable [26], but this is worthy of further investigation.

Overall, then, the low energy effective field theory approach leads to a clear understanding of the exponentially large volume scenario. We see that the stability of the large volume solution is critically dependent on the precise structure of the Kähler potential and the dynamics responsible for cancellation of the cosmological constant. Arguably, in a broad range of circumstances, corrections to the Kähler potential may well be small enough to support a large volume solution. But in the regime where the analysis is potentially reliable, it predicts a negative cosmological constant, so additional dynamics are required. The dynamics responsible for tuning the cosmological constant seems to sustain the large extra dimension solution precisely (and only) as a consequence of this tuning.

\section{General Issues in ISB}

Models with ISB raise at least two well-known issues. The first is the flavor problem. We have nothing new to add on this subject except to note that the seeming ease with which one might generate a suitable PQ symmetry is perhaps good reason to reconsider this issue, and the various solutions (flavor symmetries, features of particular regions of the moduli space) which have been proposed.

The second issue is the moduli problem. As stressed in [27], in supersymmetry axions are inevitably associated with a cosmological moduli problem, which is parametrically more severe than the usual axion energy density problem. There are basically two proposed solutions. First, moduli might sit at enhanced symmetry points. But this is incompatible with the moduli partners as axions. Second, the moduli might be sufficiently massive that the temperature after their decays is high enough to restart nucleosynthesis $[9,10]$. This corresponds to moduli masses (and presumably the gravitino mass) larger 
than $10 \mathrm{TeV}$. As usual, this poses a fine tuning problem (conceivably one whose solution could be understood anthropically). One also must account for limited production of stable particles (LSP's) in moduli decays. But it has the effect that it significantly relaxes the constraint on the axion decay constant, readily allowing axion decay constants of $10^{14} \mathrm{GeV}$ or perhaps somewhat larger, without invoking a small misalignment angle.

Related to these questions is the question of overshoot [11]. Models of the type discussed here will suffer from this difficulty; perhaps the most plausible solution is a modest tuning of initial conditions, as discussed in [28]. This tuning might plausibly have an anthropic origin. One can ask whether the problem takes a different form in the presence of multiple moduli. There are now several fields each of whose initial conditions must, to a similar degree, be tuned. The severity of this tuning might disfavor the "axiverse" scenario [15]. On the other hand, in a landscape, it is conceivable that there are many

more "states" with larger numbers of Kähler moduli, and this effect might overwhelm the effects of tuning, favoring an axiverse. These issues will be discussed in a subsequent publication [29].

\section{Axions in Low Scale (Gauge Mediation) and High Scale Su- persymmetry Breaking}

By low scale supersymmetry breaking, we mean $m_{3 / 2} \ll \mathrm{TeV}$. Generally, for such low scales, assuming Planck scale couplings for the moduli as would naively arise in the KKLT scenario, for example, these fields are cosmologically problematic. They quickly come to dominate the energy of the universe, and at the same time they are quite light, decaying long after nucleosynthesis.

So if supersymmetry is broken at low scales, then we have to assume that, in fact, there are no light moduli at scales just below the fundamental scale. In that case, any would-be axions must arise from a Peccei-Quinn symmetry which is linearly realized below the fundamental scale. This possibility is explored in [12]. It is not difficult to write such models, accounting for the quality of the Peccei Quinn symmetry through discrete symmetries. These symmetries, however, are rather intricate and it is not clear why such a structure would be generic. In this framework, it is natural for $f_{a}$ to be $10^{11}-10^{12}$ $\mathrm{GeV}$, as this scale is readily connected to some messenger scale, and also because it tends to ameliorate the quality problem.

This framework avoids the ISB moduli problem, and, assuming a gauge mediated structure, also avoids problems of flavor changing currents. But the existence of a high quality PQ symmetry is a puzzle.

Given our limited knowledge of string theories without supersymmetry, we can consider, instead, possible vacua in which the supersymmetry breaking is well below the fundamental scale, but not so low as to resolve the hierarchy problem. We can again take KKLT as a model for moduli stabilization. There seems no difficulty in accounting for an axion in this framework. Needless to say, the flavor problems of 
ISB are alleviated in such a regime, as are the cosmological issues connected with moduli. So axions would seem particularly plausible in the absence of low energy supersymmetry. But as the supersymmetry breaking scale approaches the fundamental scale, the problem of the axion quality becomes progressively more severe, suggesting that some degree of low energy supersymmetry might have something to do with the solution of the strong $\mathrm{CP}$ problem.

\section{Outlook and Scorecard}

String theory provides the most promising setting for the PQ solution of the strong CP problem. We have seen that:

1. In theories with approximate supersymmetry below the fundamental scale, the superpartners of would-be axions must be fixed by supersymmetry breaking dynamics.

2. In the small $W_{0}$ (or racetrack or Kähler stabilization) scenario(s), with multiple Kähler moduli, one can readily understand the presence of axions of high quality, from quite generic low energy effective actions.

3. In the small $W_{0}$ scenario, these additional moduli can readily, themselves, be responsible for supersymmetry breaking.

4. ISB suffers from the standard problems of flavor and cosmological moduli. The Brustein-Steinhardt problem may be more severe in such a picture.

5. In low energy supersymmetry breaking, any PQ symmetry (responsible for the QCD axion and possibly other light pseudoscalars) should be linearly realized below the fundamental scale; the breaking should be visible within the low energy theory. Flavor and moduli are not severe problems, but understanding the quality of the PQ symmetry is more challenging than in IMB.

6. As a model for the absence of low energy supersymmetry, we can simply take the supersymmetry breaking scale large (tuning the weak scale); suitable axions remain highly plausible, and many of the problems of ISB are ameliorated.

¿From these observations, it seems that axions are a quite plausible outcome of a landscape picture for understanding the laws of nature. ISB seems the most plausible setting. If the Peccei-Quinn symmetry is non-linearly realized below the fundamental scale, it is hard to see how to adequately protect the axion without low energy (but not necessarily weak scale) supersymmetry. If it is linearly realized, either in models of low energy supersymmetry breaking or in models without low energy supersymmetry, intricate discrete (gauged) symmetries seem required to account for the high degree of axion quality. 
Acknowledgements Conversations with Tom Banks and Leonard Susskind are gratefully acknowledged. This work was supported in part by the U.S. Department of Energy. M. Dine thanks Stanford University and the Stanford Institute for Theoretical Physics for a visiting faculty appointment while much of this work was performed. J. Kehayias thanks Mark Goodsell for useful conversations and references while at Susy10. G. Festuccia is supported by NSF grant NSF PHY-0969448. Any opinions, findings, and conclusions or recommendations expressed in this material are those of the authors and do not necessarily reflect the views of the National Science Foundation.

\section{References}

[1] E. Witten, "Some Properties of O(32) Superstrings," Phys.Lett. B149 (1984) 351-356.

[2] S. Kachru, R. Kallosh, A. D. Linde, and S. P. Trivedi, "De Sitter vacua in string theory," Phys. Rev. D68 (2003) 046005, arXiv:hep-th/0301240.

[3] T. Banks, M. Dine, and E. Gorbatov, "Is there a string theory landscape?," JHEP 0408 (2004) 058, arXiv:hep-th/0309170 [hep-th].

[4] J. F. Donoghue, "Dynamics of M theory vacua," Phys.Rev. D69 (2004) 106012, arXiv:hep-th/0310203 [hep-th].

[5] J. P. Conlon, F. Quevedo, and K. Suruliz, "Large-volume flux compactifications: Moduli spectrum and D3/D7 soft supersymmetry breaking," JHEP 08 (2005) 007, arXiv:hep-th/0505076.

[6] K. Choi and K. S. Jeong, "String theoretic QCD axion with stabilized saxion and the pattern of supersymmetry breaking," JHEP 01 (2007) 103, arXiv:hep-th/0611279.

[7] B. S. Acharya, K. Bobkov, and P. Kumar, "An M Theory Solution to the Strong CP Problem and Constraints on the Axiverse," arXiv:1004.5138 [hep-th].

[8] K. Bobkov, V. Braun, P. Kumar, and S. Raby, "Stabilizing All Kahler Moduli in Type IIB Orientifolds," arXiv:1003.1982 [hep-th].

[9] T. Banks, D. B. Kaplan, and A. E. Nelson, "Cosmological Implications of Dynamical Supersymmetry Breaking," Phys. Rev. D49 (1994) 779-787, arXiv:hep-ph/9308292.

[10] B. de Carlos, J. A. Casas, F. Quevedo, and E. Roulet, "Model independent properties and cosmological implications of the dilaton and moduli sectors of 4-d strings,"

Phys. Lett. B318 (1993) 447-456, arXiv:hep-ph/9308325.

[11] R. Brustein and P. J. Steinhardt, "Challenges for superstring cosmology," Phys. Lett. B302 (1993) 196-201, arXiv:hep-th/9212049.

[12] L. M. Carpenter, M. Dine, G. Festuccia, and L. Ubaldi, "Axions in Gauge Mediation," Phys.Rev. D80 (2009) 125023, arXiv:arXiv:0906.5015 [hep-th]. 
[13] M. Dine, "The intermediate scale branch of the landscape," JHEP 01 (2006) 162, arXiv:hep-th/0505202.

[14] K. Choi, A. Falkowski, H. P. Nilles, and M. Olechowski, "Soft supersymmetry breaking in KKLT flux compactification," Nucl. Phys. B718 (2005) 113-133, arXiv:hep-th/0503216.

[15] A. Arvanitaki, S. Dimopoulos, S. Dubovsky, N. Kaloper, and J. March-Russell, "String Axiverse," Phys. Rev. D81 (2010) 123530, arXiv:0905.4720 [hep-th].

[16] R. Bousso and J. Polchinski, "Quantization of four form fluxes and dynamical neutralization of the cosmological constant," JHEP 0006 (2000) 006, arXiv:hep-th/0004134 [hep-th].

[17] T. Banks and M. Dine, "Coping with strongly coupled string theory," Phys.Rev. D50 (1994) 7454-7466, arXiv:hep-th/9406132 [hep-th].

[18] M. Dine, J. L. Feng, and E. Silverstein, "Retrofitting O'Raifeartaigh models with dynamical scales," Phys. Rev. D74 (2006) 095012, arXiv:hep-th/0608159.

[19] M. Dine and J. Kehayias, "Discrete R Symmetries and Low Energy Supersymmetry," Phys. Rev. D82 (2010) 055014, arXiv:0909.1615 [hep-ph].

[20] P. Breitenlohner and D. Z. Freedman, "Positive Energy in anti-De Sitter Backgrounds and Gauged Extended Supergravity," Phys. Lett. B115 (1982) 197.

[21] P. Breitenlohner and D. Z. Freedman, "Stability in Gauged Extended Supergravity," Ann. Phys. 144 (1982) 249.

[22] M. Berg, M. Haack, and E. Pajer, "Jumping Through Loops: On Soft Terms from Large Volume Compactifications," JHEP 0709 (2007) 031, arXiv:arXiv:0704.0737 [hep-th].

[23] M. Cicoli, J. P. Conlon, and F. Quevedo, "Systematics of String Loop Corrections in Type IIB Calabi-Yau Flux Compactifications," JHEP 0801 (2008) 052, arXiv:arXiv:0708.1873 [hep-th].

[24] J. Kehayias and W. Wu. To appear.

[25] R. Blumenhagen, J. P. Conlon, S. Krippendorf, S. Moster, and F. Quevedo, "SUSY Breaking in Local String/F-Theory Models," JHEP 09 (2009) 007, arXiv:0906.3297 [hep-th].

[26] J. P. Conlon and E. Palti, "On Gauge Threshold Corrections for Local IIB/F-theory GUTs," Phys. Rev. D80 (2009) 106004, arXiv:0907.1362 [hep-th].

[27] T. Banks, M. Dine, and M. Graesser, "Supersymmetry, axions and cosmology," Phys. Rev. D68 (2003) 075011, arXiv:hep-ph/0210256.

[28] B. Freivogel, M. Kleban, M. Rodriguez Martinez, and L. Susskind, "Observational consequences of a landscape," JHEP 03 (2006) 039, arXiv: hep-th/0505232.

[29] W. Wu. To appear. 